\title{
Formal Support for the ELLA Hardware Description Language
}

\author{
Howard Barringer Graham Gough Brian Monahan ${ }^{\star}$ Alan Williams ${ }^{\star \star}$ \\ University of Manchester, UK $\mathrm{UK}^{\star \star \star}$ \\ email: howard@cs.man.ac.uk
}

\begin{abstract}
We describe the development of formal verification support tools for the commercial hardware description language ELLA, which are embedded into an industrial-style hardware design system, to be utilised by hardware engineers. A formal semantics for ELLA is given using various semantic representations, including state machines and process algebraic terms, so that different formal analysis methods can be used. In particular, a novel symbolic verification method can be used with the process terms generated from ELLA-text, giving a high level and efficient means of verifying the correctness of ELLA designs.
\end{abstract}

Keywords: hardware design tools, formal semantics, symbolic verification, ELLA.

\section{Introduction}

This paper presents an overview of work on providing formal verification support for the commercial hardware description language ELLA[1]. We describe our approach to defining the formal semantics of ELLA, and how the resulting definitions are used as a basis for the implementation of compilers, simulators, and various formal verification tools. These are all embedded in a conventional commercial-style hardware design environment. The emphasis in our approach has been to provide formal analysis tools which operate largely automatically for commercially sized designs, and with which the designer can interact at the level of the source hardware description language (HDL). Using such a system, the designer can integrate formal analysis techniques into current design methodologies, and does not have to be concerned with the underlying formal semantic foundations.

This work has resulted in a collection of denotational semantic definitions [2] for ELLA, providing the basis for associated formal verification methods. We reported our first results for providing a state-based semantics and automatic verification tools for a subset of ELLA, restricted to the booleans, at CHARME'91 [3]. We have since developed a process algebraic semantics for Core ELLA, using ELLA Process Algebra (EPA). EPA terms

\footnotetext{
* Brian Monahan is now with Harlequin Ltd., Alderley Edge, UK.

$\star \star$ Alan Williams acknowledges the additional financial support of DRA, Malvern, UK, and Harlequin Ltd, Cambridge, UK.

$\star \star \star$ The University of Manchester acknowledges support from the SERC via research grant GR/F 38174.
} 
generated from ELLA can be translated into symbolic deterministic machines, which form the basis for semi-automatic symbolic verification based on a novel state evolution rule. This establishes the standard (strong) bisimulation equivalence[4] of the machines under analysis, by generating simple logical expressions characterising the bisimulation relation. The expressions are validated separately using a standard theorem-proving tool - currently we use the PVS proof system[5]. The approach enables abstract high-level verification of designs, and can be applied to systems with infinite state-spaces.

The symbolic machine format for the semantic objects representing ELLA expressions also acts as the basis for ground and symbolic simulation tools. The formal semantics allows the derivation and proof of ELLA-level behaviour preserving rewrite rules/laws, so that a designer can manipulate a design at the ELLA level.

However, the formal analysis tools are only one part of the technology required to increase the overall reliability of hardware design - even if design components can be verified, there are other aspects of the design process which can lead to error. Therefore, a rigorous design methodology is also required, with tool support for tasks such as design management, version control, recording design decisions and status, multi-user working, and animation for exercising designs and specifications. All tools are embedded within a graphical user interface, which presents the user with a standard look and feel. The formal analysis tools are therefore integrated with the conventional tools they can be utilised within the existing design process, which will then naturally evolve to take advantage of the additional capabilities offered by the new analysis tools.

A prototype implementation of an integrated hardware design system, called the ELLA Verification Environment $(E V E)$, has been developed, which contains standard design tools such as a design database, editors, and a ground level simulator. It also includes formal analysis tools for symbolic simulation, design transformation and verification. All tools use the formal semantic definitions as the basis for the internal representation of the design. This leads to close integration of the various tools, and provides a high degree of confidence that they will be self-consistent, since they are working from common semantic definitions. We have achieved encouraging results from initial use of the prototype $E V E$, both when using the formal analysis tools, and in terms of overall ease of use during design construction and simulation.

The work on developing the EVE has been conducted jointly by Harlequin Ltd. (Cambridge), DRA (Malvern), and Manchester, as part of a collaborative project on verification support for hardware design. GEC Plessey Semiconductors (Swindon) acted as project reviewers. An overview of the work from the project appears in $[6]^{1}$. Details of the symbolic verification methods used in the $E V E$ are given in [7].

\subsection{Overview of Paper}

In the next section, we give a brief overview of ELLA, and describe the first approach used for providing a state-based formal semantics using IO automata (IOAs), together

${ }^{1}$ Reports from the ELLA Project are available over the World-Wide Web, URL: http: //www.cs.man.ac.uk/fmethods/projects/ella-project.html 
with the accompanying verification tools, and their implementation. In $\S 3$ we describe how the level of representation in the semantics is raised by using EPA. The associated symbolic verification method, based on the state evolution rule, is described in $\S 4$. Finally in $\S 5$ we present several design examples that highlight the various characteristics of each verification approach, and further discuss implementation issues of the prototype design environment.

\section{State-based Semantics of ELLA}

ELLA is a commercial hardware description language developed over a number of years by the Royal Signals and Radar Establishment at Malvern[1 $]^{2}$. A commercial design system for ELLA is available, including compilation and simulation tools $[8]^{3}$. The language has been specifically designed so that complex ELLA constructs can be translated into equivalent ELLA expressions, containing only constructs taken from a covering Core subset. ELLA also has a simple timing model. These characteristics somewhat ease the task of providing a formal semantic definition - it is possible to consider the formal semantics of the Core which, together with the Full-to-Core transformations, gives a semi-formal semantics covering full ELLA. The language also supports complex datatypes, including enumerated types, compound types, and finite integer ranges, with built-in arithmetic operations available on the latter. We refer the reader to [1] for further details.

ELLA does not offer the wide range of constructs provided by VHDL[9]. However, this relative simplicity, coupled with its simple timing model, has the distinct advantage that an intuitive state-based semantics can be defined, significantly easing the task of checking that the formal semantics coincides with the informal meaning intended by the language designers.

As an example of ELLA, the following text describes a simple Delay-NOR gate; this will be used below to illustrate the main semantic representations defined compositionally for ELLA:

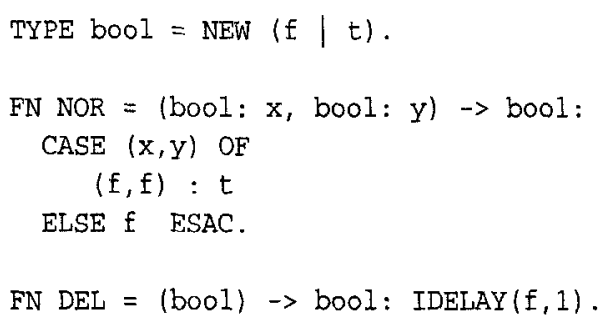

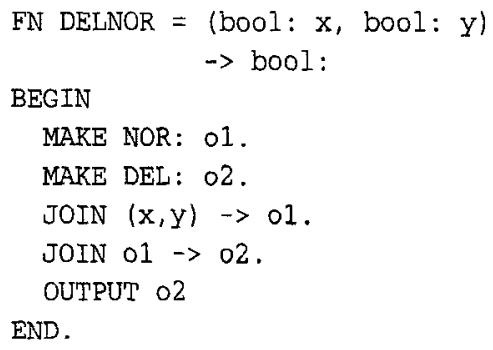

\footnotetext{
${ }^{2}$ RSRE Malvern became the Defence Research Agency (Malvern), and is referred to in the rest of the paper as DRA.

${ }^{3}$ ELLA is now marketed by R-Cube Systems, Bristol, England (email parer-cube.demon.co.uk), and the public-domain version (ELLA2000) is available by anonymous ftp from Imperial College, London, in src.doc.ic.ac.uk:packages/ELLA
} 


\subsection{IO Automaton Semantics}

The semantic definition for ELLA is developed in stages. First, the covering Core subset of ELLA is identified, with the intention of providing semantic definitions for this only; a suite of software transformations are implemented ${ }^{4}$, so that complex (full) ELLA constructs can be translated into equivalent Core ELLA expressions. This reduces the size of the semantic definition somewhat. However, the syntax of Core ELLA is still quite large and therefore we first consider a subset called Kernel ELLA, with a further (formally defined) translation from Core to Kernel - we can still represent all ELLA expressions using this, due to the Full-to-Core and Core-to-Kernel transformations. Initially we also restrict to boolean types only, and produce a semantics for Boolean Kernel ELLA $(B K E)$, which is a strict subset of ELLA.

The basic semantics of Core ELLA is given as a set of input/output traces, each element containing a pair of maps, with maplets from channel names to values. We represent the semantics as Input/Output automata (IOAs), similar to the structures described in [10], because they are well-understood, and there are established methods for determining behavioural equivalence. The simultaneous input/output behaviour of ELLA is captured by the i/o map pairs labelling the transitions in the IOAs, binding values to channel names. The synchronous behaviour of composite ELLA expressions is then determined by defining a special product operation between the IOA objects representing the components. The resulting product IOA only produces a transition when each of the components can make a transition, ensuring that the values on same-name channels are in agreement. Unwanted output channels can be removed from the transition labels, and channels names can be changed, using, respectively, hiding and renaming operations on the IOAs.

The semantics of ELLA is given by a valuation function taking ELLA expressions to IOA objects, defined over the structure of the ELLA syntax. Basic ELLA expressions - for example signals, constants, unit delay, or case statements - are represented by predefined atomic IOAs. The valuation function then determines how these are to be composed in order to represent the behaviour of complex ELLA constructs, using the product, hiding and renaming operations on IOAs. An environment is used to store intermediate results, such as the objects representing previously defined ELLA functions.

For example, Table 1 shows the IOA transition relations representing the behaviour of the ELLA functions NOR, DEL, and DELNOR given above. IOA (c) is obtained from (a) and (b) by using the product operation, effectively 'connecting' the output $o 1$ of the NOR Gate, to the same-label input of the unit delay, and including only those transitions in the final IOA where the values on channel $o 1$ are in agreement. The hiding operation is then used to remove the output channel $o 1$. The new state labels are taken directly from IOA (b), because (a) is a single-state machine. Note that the unit delay IOA is atomic, but the NOR gate IOA will have been built up from its component IOAs, representing constants, signals, case and tupling. Notice also how the resulting object is low-level and bears little resemblance to the original text; any structure in the original ELLA description of DELNOR has now been lost.

${ }^{4}$ The software transformations, taking (full) ELLA constructs into equivalent Core ELLA expressions, are part of the ELLA2000 system developed by DRA, Malvern. 


\begin{tabular}{|c|c|c|c|c|}
\hline From & Input & Output & To \\
\cline { 2 - 4 } State & x & y & o1 & State \\
\hline n & f & $f$ & $t$ & $n$ \\
n & $f$ & $t$ & $f$ & $n$ \\
n & $t$ & $f$ & $f$ & $n$ \\
n & $t$ & $t$ & $f$ & $n$ \\
\hline
\end{tabular}

(a)

\begin{tabular}{|c|c|c|c|}
\hline From & Input & Output & To \\
\cline { 2 - 3 } State & o1 & o2 & State \\
\hline 0 & $\mathrm{f}$ & $\mathrm{f}$ & 0 \\
1 & $\mathrm{f}$ & $\mathrm{t}$ & 0 \\
0 & $\mathrm{t}$ & $\mathrm{f}$ & 1 \\
1 & $\mathrm{t}$ & $\mathrm{t}$ & 1 \\
\hline
\end{tabular}

(b)

\begin{tabular}{|c|c|c|c|c|}
\hline From & Input & Output & To \\
\cline { 2 - 4 } State & $\mathrm{x}$ & $\mathrm{y}$ & $\mathrm{o}$ & State \\
\hline 0 & $\mathrm{f}$ & $\mathrm{f}$ & $\mathrm{f}$ & 1 \\
0 & $\mathrm{f}$ & $\mathrm{t}$ & $\mathrm{f}$ & 0 \\
0 & $\mathrm{t}$ & $\mathrm{f}$ & $\mathrm{f}$ & 0 \\
0 & $\mathrm{t}$ & $\mathrm{t}$ & $\mathrm{f}$ & 0 \\
1 & $\mathrm{f}$ & $\mathrm{f}$ & $\mathrm{t}$ & 1 \\
1 & $\mathrm{f}$ & $\mathrm{t}$ & $\mathrm{t}$ & 0 \\
1 & $\mathrm{t}$ & $\mathrm{f}$ & $\mathrm{t}$ & 0 \\
1 & $t$ & $t$ & $t$ & 0 \\
\hline
\end{tabular}

(c)

Table 1. IOA Transition Tables: (a) NOR Gate, (b) Unit Delay, (c) Delay NOR Gate

\subsection{Design Analysis and Tool Implementation}

The IOAs generated by the Core-to-IOA compilation tools can now be analysed using standard trace-equivalence and model-checking techniques[3]. The trace-equivalence checker generates a counter-example machine when two designs are inequivalent. This provides the designer with traces leading to differences in behaviour, which may be useful during debugging; however, the error traces generated may not always be helpful to the designer in determining the source of the error - for example, a single error may lead to a large number of error traces. Analysis tools from a related research project are also used for checking temporal properties, displaying and animating the IOAs, and for checking design equivalence using BDDs (limited to a combinatorial subset only in the prototype). Results are given in $\$ 5$ below.

A prototype of the compilation, simulation and verification tools based on the formal semantic definitions, is implemented in Common Lisp, and embedded into a hardware design environment, containing text and schematic editors, design database, and a soft logic analyser for visualising input and output of simulations, and equipped with a sophisticated graphical user interface[11] $]^{5}$. The compositional style of the semantic definitions means that a Common Lisp implementation is straightforward, with there being a close correspondence between the definitions and resulting code in the Core ELLAto-IOA compiler.

5 The environment was developed jointly by the ELLA Project partners, using the LispWorks programmer's environment[12], which is produced by, and a trademark of Harlequin Ltd. (Cambridge, UK). Harlequin developed the environment framework and tools, DRA implemented the ELLA-to-Kernel ELLA parsing and compilation tools, and Manchester implemented the Kernel ELLA-to-IOA compiler, and associated IOA analysis tools. 
Even though these state-based tools are first-cut prototypes, they will still be able to play a role in the final design system - for certain small designs, state-based verification approaches may be the most efficient. At the very least, we can now easily compare various verification methods, simply by switching the back-end semantic objects generated by the compiler, from the same ELLA-text. Additionally, the IOA representation facilitates interfacing with other external state-based analysis tools, as they become available.

\subsection{Discussion}

IOAs were chosen initially to represent the semantics of ELLA because they are wellunderstood, and there are associated standard automatic equivalence-checking methods, with counter-examples generated if two designs are found to be inequivalent. It is also possible to represent temporal properties as IOAs, and check that a design satisfies these properties. We were therefore free at this stage to concentrate on establishing the basic approach, in particular ensuring that the formal semantics coincided with the informal meaning inferred by the standard ELLA compilation and simulation tools, and with the intuitions of the designers of ELLA at DRA. Prototype tools could also be implemented quickly, so that we could exercise the resulting semantic objects.

The (formal) meaning of the ELLA expressions was determined by using a combination of sources: the ELLA language documentation, commercial design and simulation tools [8], and extensive discussions with the language designers. After the prototype tools for compilation, simulation and verification had been implemented, based on this semantic definition, further comparisons and reviews were undertaken, with the significant advantage of being able to use the same ELLA-text source for the prototype and commercial design systems. This development also served to clarify ELLA language issues in the existing commercial and public domain tools ${ }^{6}$.

Of course, due to the problems of state- or transition-explosion, even with binary decision diagram (BDD) encodings, we planned later to develop higher level representations. The IOA representation also operates at a low-level - it is not straightforward to build design transformation tools based on IOA representations, and the debugging feedback is given in low-level terms, as sets of differentiating traces. The representation is flattened, and all of the initial design structure has been removed by the compilation process, thus making the process of utilising the debugging information that much harder — the designer must analyse ground-value error-traces in order to find problems in a system.

Therefore we proceed to modify the semantics, both to include the full typing structures of ELLA (and therefore provide a full formal semantics for Core ELLA), and also to address the problems of representing the semantics efficiently and abstractly. This is carried out in two stages; first, the full type structures from Core ELLA are included in the IOA semantics, by a straightforward extension.

\footnotetext{
${ }^{6}$ Arising from this close scrutiny of the existing tools, a minor inconsistency was also later uncovered between the commercial and public domain compilation and simulation tools for ELLA, involving the value obtained during simulation when defining a constant integer via an ARITH function.
} 
Next, we consider a high-level algebraic representation of the semantics. The 'layered' IOA semantic definition means that it is relatively straightforward to replace the semantics objects with an alternative representation, with appropriately defined operators on the semantic objects, re-using the semantic definitions which are built on top of these. This reduces the amount of work required in reformulating the semantics, but most significantly, it helps to reduce the chances of introducing errors into the semantic definitions during the refinement.

Our aim is to provide the designer with formal verification tools which can operate efficiently at a high-level, in terms of the initial HDL, so that, for example, debugging information is produced as fragments of the original designs. We also wish to be able to apply transformations on the semantic objects, both for design manipulation and in order to simplify the semantic objects during processing.

Full details of the IOA semantics for $B K E$, together with trace-equivalence and temporal property checking, appear in [3]; the complete IOA semantics for Core ELLA appears in [13].

\section{Process Algebraic Semantics}

There are already several well-established process algebras for modelling concurrent systems, such as CSP[14], SCCS[4], CIRCAL[15] and LOTOS[16]. It would be possible to model the semantics of ELLA within one of these. However we wished to avoid this overhead, and to give a direct semantics, in particular giving direct process algebraic expressions corresponding to the richly structured i/o maps and product operation for IOAs. We therefore developed the process algebra $E P A$, which can directly represent IOAs at an intermediate level. EPA possesses many of the characteristics of the more standard process algebras, such as an operational semantics and the usual collection of process combinators - prefix, summation, product, hiding. However, EPA separates design structure and data, by having a rich action algebra to represent functional behaviour of a design, and using the process algebraic constructors to represent design composition, and state. $E P A$ also has parameterised process calls for passing state information and channel labels. The process product operator is defined in terms of an action product which exactly captures the IOA product.

The IOA semantics for Core ELLA is now redefined, using EPA terms as the underlying semantic objects. The valuation functions defined for the IOA semantics are modified by replacing the atomic IOAs and IOA operations, with corresponding EPA process definitions and EPA product, renaming, and hiding operations. Built-in ELLA functions, such as CASE or arithmetic operators are defined directly as value expressions in the ELLA action algebra, which can be bound to channels, or appear as 'state' arguments in process calls. Certain aspects of the resulting EPA semantics for ELLA can then be simplified; for example channel renaming is now reduced to calling a particular process with the required channel name arguments, whereas previously it involved defining a special renaming IOA which was then composed with the desired semantic object.

We refer the reader to [6] for further details of EPA, including definitions of its transition- 
based semantics, and an overview of the EPA semantics for Core ELLA - the complete definition of the semantics appears in [17].

\subsection{EPA Examples}

As simple examples of EPA terms representing ELLA, consider again the NOR gate, unit-delay and delay-NOR gate from $\$ 2.1$. First, the (delay-less) NOR-gate can be represented by a process Nor $_{P}$ defined by:

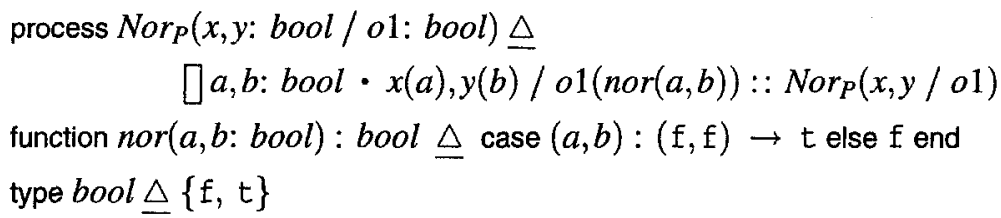

This process definition has input channels $x, y$ and output channel $o 1$ as parameters. The right-hand side contains a choice ' $\square a, b$ : bool - ' meaning that values for variables $a, b$ are chosen from the set bool. The remaining term contains a prefix action ' $x(a), y(b) / o 1($ nor $(a, b))$ ', which is invoked before the process evolves into the continuation ' $\operatorname{Nor}_{P}(x, y / o 1)$ '; this is simply a recursive call to the same process Nor $_{P}$ in this case. The action corresponds to the transition labels used in the IOA, and has input bindings ' $x(a), y(b)$ ', and output binding ' $o 1($ nor $(a, b))$ '. Here, the function expression $n o r(a, b)$ is bound to the output channel $o 1$, giving the process $\operatorname{Nor}_{P}$ its intended logical 'nor' meaning.

A unit delay can be represented by a process definition $U d e l_{[b o o l]}$ which has a 'state' variable $w$ :

$$
\begin{aligned}
& \text { process } \operatorname{Udel}_{[b o o l]}(w: \text { bool } ; \text { ol }: \text { bool } / o 2: \text { bool }) \triangle \\
& \square v: \text { bool } \cdot o 1(v) / o 2(w):: \operatorname{Udel}_{[t y]}(v ; o 1 / o 2)
\end{aligned}
$$

This process now has an extra parameter $w$ (distinguished from the channels by appearing before the separator ' ; '), which enables current 'state' value expressions to be used. At every transition, $U d e l_{[b o o l]}$ chooses a value $v \in$ bool for input channel $o 1$, and binds the current state value $w$ to output $o 2$. It then evolves into a recursive call, using the value $v$ just received on its input as the new 'state'; this will be output at the next transition, thus effecting a unit delay ${ }^{7}$.

Now to represent a unit-delay-Nor gate, the two process terms $\operatorname{Nor}_{P}$ and $U d e l_{[b o o l]}$ must be combined to evolve in synchrony using a synchronous process composition operator ' $\mid$ '. A common channel $o 1$ is introduced for the output of Nor $_{P}$ and the input of $\operatorname{Udel}_{[b o o l]}$, which is then hidden, indicated by the part of the term ' $(\cdots) \backslash\{o 1:$ bool $\}$ '.

\footnotetext{
${ }^{7}$ In the $E P A$ semantics, a generic unit delay process definition is defined instead, parameterised by type, and then instantiated for each concrete type. Essentially the same compact EPA term is used for any type, while the corresponding (maximally connected) IOA would have $k$ states, and $k(k-1)$ transitions, where $k$ is the cardinality of the type.
} 
process $\operatorname{DelNor}_{P}(v$ : bool ; $x, y$ : bool / o2: bool $) \triangle$

$$
\left(\operatorname{Nor}_{P}(x, y / o 1) \mid \operatorname{Udel}_{[b o o l]}(v ; o 1 / o 2)\right) \backslash\{o 1: b o o l\}
$$

At each step, the actions from the component processes are combined with an action product operator ' $\star$ ', which forms a valid composite action only when the values bound to like channel names (i.e. o1) are in agreement, similar to the corresponding IOA product operation on $\mathrm{i} / \mathrm{o}$ maps.

With the new EPA semantics, it is now possible to reason algebraically about the highlevel structured semantic objects generated from ELLA. In particular, simplification rules for both process terms and action terms can be applied during compilation, for example, to eliminate 'synonym' channels, or paired tuple/projection expressions (e.g. the expression ' $\left(x_{1}, x_{2}\right)[2]$ ' simplifies to ' $x_{2}$ '). Nested hiding can also be removed, so that the compiler generates process terms with the following form:

$$
\left(P_{1}|\cdots| P_{k}\right) \backslash\left\{c_{1}, \cdots, c_{m}\right\}
$$

with process calls $P_{i}$, and hidden channels $c_{j}$. This helps to further improve the efficiency of the compilation tools, by reducing the size of intermediate terms generated.

\section{Formal Analysis}

Any EPA term generated from ELLA, such as that above, can be further transformed into an equivalent symbolic ' deterministic machine' form:

$$
P\left(v: S t_{P} ; i: I / o: o\right) \triangleq \square x: I \cdot i(x) / o\left(r_{P}(x, v)\right):: P\left(e_{P}(x, v) ; i / o\right)
$$

where $P$ has a single state parameter $v$ of type $S t_{P}$ (the 'state-space' of $P$ ), an input $i$ of type $I$, and an output $o$ of type $O$. The output value is given by a response function expression $r_{P}$, and the next state by an evolution function expression $e_{P}$. The choice $x: I$ binds the variables occurring in the input actions. The initial starting state for $v$ is taken as $i_{P} \in S t_{P}$. The deterministic machine $D_{P}$ for process $P$ is given by the following triple:

$$
\begin{aligned}
& D_{P}=\left\langle i_{P}, r_{P}, e_{P}\right\rangle \\
& \text { where } \\
& i_{P} \in S t_{P} \\
& \text { - initial state } \\
& r_{P}:\left(I \times S t_{P}\right) \rightarrow O \\
& \text { - response function } \\
& e_{P}:\left(I \times S t_{P}\right) \rightarrow S t_{P} \\
& \text { - evolution function }
\end{aligned}
$$

For example, the process definition given above for DelNor $_{P}$ can be translated into the following deterministic machine form:

$$
\begin{aligned}
& \text { process DelNor }(v ; x, y: \text { bool / o2: bool }) \unlhd \\
& \square a, b: \text { bool } \cdot x(a), y(b) / o 2(v):: \operatorname{DelNor}_{P}(\operatorname{nor}(a, b) ; x, y / o 2)
\end{aligned}
$$


where the output gives the current state value $v$, and the next state is now given by the value expression $\operatorname{nor}(a, b)$.

The deterministic machine extracted from the simplified EPA term compiled from ELLA, forms the basis of simulation and verification tools. For an efficient method of ground level simulation, the raw EPA compiler output is translated into Lisp code which can then be compiled using the standard Lisp compiler, and then executed ${ }^{8}$.

\subsection{Symbolic Simulation}

Symbolic simulation is essentially another application of the simplification rules developed for the EPA compiler. A graphical user interface (GUI) enables the user to specify input expressions, and to select which parts of the design are displayed or expanded, so that there is control over the potential growth in size of expression as a symbolic simulation evolves. The expressions can be displayed to the user in an ELLA syntax, because EPA is able to express the structure in the design, which can been retained during compilation. The symbolic simulator can be used to explore signal flow through a circuit, showing how the value expressions on circuit nodes evolve through time. We refer the reader to [18] for further details.

\subsection{Symbolic Verification}

We have developed a novel state evolution method for establishing that two deterministic machines are equivalent. This effectively performs a symbolic simulation of the two machines for a finite number of steps, and generates a set of simple (first-order) verification conditions (VCs) which, if valid, show that the two machines are behaviourally equivalent. The user must supply the number of steps, $N$, which is usually related to the latency or testability of the designs. The validity of the generated VCs can be checked using a separate theorem-proving tool — at present the PVS system is used[5]. The task of verification has therefore been broken down into two separate operations: one of generating the set of VCs from the ELLA design descriptions, and then proving their validity. This separation allows the designer to add extra information, in the form of axioms, into the theory presented to the theorem-prover - see the examples below, which illustrate this.

For certain designs, it may not be possible to prove the validity of the verification conditions in a finite number of steps, due to the fundamental undecidability of the problem. However, for designs $P$ and $Q$, if the product machine $D_{P} \times D_{Q}$ has bounded history that is, after a finite number of steps, the output does not depend on the initial state then a finite $N$ can be found, and the state evolution rule will be complete.

${ }^{8}$ For example, the specification of a 6502 microprocessor, consisting of 1600 lines of ELLA can be simulated at approximately 20 processor cycles per second, using the ground level simulator developed by Harlequin Ltd. 
4.2.1 State Evolution At stage $N \geq 1$ in the method, we compare the responses, based upon the previous $N-1$ steps. The relation $R_{N}$ holds if the responses for two machines $P, Q$ are in agreement at time step $N$, for all input sequences $x_{1}, \cdots, x_{N} \in I$ having started at time 0 from arbitrary states $\sigma_{P} \in S t_{P}$ and $\sigma_{Q} \in S t_{Q}$ :

$$
\begin{aligned}
& R_{N}\left(\sigma_{P}, \sigma_{Q}\right) \Leftrightarrow \forall x_{N}, \cdots x_{1} \cdot r_{P}\left(x_{N}, s p_{N}\right)=r_{Q}\left(x_{N}, s q_{N}\right) \\
& \text { where } \\
& \quad \forall i \in\{2, \ldots, N\} \cdot s p_{i}=e_{P}\left(x_{i-1}, s p_{i-1}\right) \wedge s q_{i}=e_{Q}\left(x_{i-1}, s q_{i-1}\right) \\
& \quad \wedge s p_{1}=\sigma_{P} \wedge s q_{1}=\sigma_{Q}
\end{aligned}
$$

An 'accumulating' version of $R$ holds if the responses of $P$ and $Q$ are in agreement, at all time-steps, again for all input sequences, starting from states $\sigma_{P}, \sigma_{Q}$ :

$$
A R_{N}\left(\sigma_{P}, \sigma_{Q}\right) \Leftrightarrow \bigwedge_{i=1}^{N} R_{i}\left(\sigma_{P}, \sigma_{Q}\right)
$$

Using these relations, we can define a state evolution rule, which says that if we can establish for some fixed $N$ :

(a) the machines have equivalent responses for $N$ steps starting from their initial state pair $\left\langle i_{P}, i_{Q}\right\rangle$, and

(b) on the assumption that the machines have equivalent responses for $N$ steps starting from arbitrary state pairs $\left\langle\sigma_{P}, \sigma_{Q}\right\rangle$, we can infer that their responses are equivalent for step $N+1$

we can conclude that the two deterministic systems $P$ and $Q$ are equivalent.

\section{Definition 1 : State Evolution Rule}

For some fixed $N \in \mathbb{N}$ :

$$
\text { SE } \begin{aligned}
& \text { (a) } \Gamma \vdash A R_{N}\left(i_{P}, i_{Q}\right) \\
& \text { (b) } \Gamma \vdash \forall \sigma_{P}, \sigma_{Q} \cdot A R_{N}\left(\sigma_{P}, \sigma_{Q}\right) \Rightarrow R_{N+1}\left(\sigma_{P}, \sigma_{Q}\right)
\end{aligned}
$$

where ' $P \sim_{S B} Q$ ' denotes that the deterministic machines $P$ and $Q$ are behaviourally equivalent - the process terms from which they were derived are therefore strongly bisimilar [4]. The theory $\Gamma$, apart from containing process definitions, may also contain design assumptions in the form of axioms. See [7] for a detailed account. 
Note in particular that the initial premise (a) is valid if the two designs have equal outputs for $N$ steps, when starting from the initial state pair $\left\langle i_{P}, i_{Q}\right\rangle$ of the machines for all input sequences. Step premise (b) is valid if it can be inferred that the designs have equal outputs at the $N+1$ th step, on the assumption that they have equal outputs for the previous $N$ steps, where the symbolic simulation starts from arbitrary states $\left\langle\sigma_{P}, \sigma_{Q}\right\rangle$.

4.2.2 The Verification Condition Generator (VCG) The expressions for the response and evolution functions, which are instantiated in the two logical expressions for the premises of the state evolution rule, have case statements as their control structures, where the guard sets for each case arm are disjoint and covering. Using this information, each premise can be decomposed into a set of VCs, whose conjunction is equivalent to the original premise. A VC has a regular structure, where the conclusion is an equality, and the premise is a conjunction of constraints and equalities, as follows:

$$
\begin{aligned}
& \left(\left(\text { constr }_{1} \wedge \cdots \wedge \text { const }_{k}\right) \wedge\left(\text { equality }_{1} \wedge \cdots \wedge \text { equality }_{m}\right) \Rightarrow \text { concl }\right) \\
& \text { where } \\
& \forall i \in\{1, \ldots, k\} \cdot \text { constr }_{i}=\left(\text { var }_{i} \in \text { set }_{i}\right) \\
& \forall i \in\{1, \ldots, m\} \cdot \text { equality }_{i}=\left(\text { lhs-expr } r_{i}=r h s-\operatorname{expr}_{i}\right) \\
& \text { concl } \left.=\left(\text { concl-expr } r_{1}=\text { concl-expr }\right)_{2}\right)
\end{aligned}
$$

All variables are universally quantified over appropriate data sets. The constraints are derived from the guards in the various case statements within the response and evolution functions, and the conclusion gives the required equality between machine outputs, at some stage in their symbolic simulation. Therefore the elements in each VC can be related back directly to fragments of the original designs. The simple structure facilitates checking that each VC is valid, which can be significantly automated, using a theoremprover/proof assistant such as PVS[5]. An example of the VCs generated for a simple integer latch design is given below in $\S 5(3)$.

During VC generation, the VCG can perform extensive simplification on the VCs, and discard those that can be found to be true, therefore reducing the final number of VCs produced. Without any simplification, the number of VCs generated would be equal approximately to the product of the case branching sizes of the two machines for each symbolic simulation step, since it would not be possible, for example, to identify VCs containing contradictory guard sets. The VCG therefore applies set expression simplification rules which attempt to identify empty sets, indicating contradictory guards and thus a valid VC, without expanding the set expressions.

It is also possible to perform functional abstraction during verification, by not expanding function definitions, and then possibly providing additional axioms characterising the behaviour of these functions at the theorem-proving stage, needed to be able to prove the VCs. This may well reduce the complexity and number of VCs generated. For example, the latch design given in $\S 5$ only requires a standard div/mod property of integer arithmetic in order to validate the VCs, and therefore prove the designs equivalent. In the FIR filter design example, only the commutative/associative properties of addition 
and multiplication are needed. Additionally the VCG may be able to eliminate identical control branches from the two designs. These techniques can therefore significantly reduce the verification task, by exploiting structural similarities in the two designs under analysis.

4.2.3 Discussion The state evolution rule defines a pair of first-order logical expressions which, if valid, characterise the equivalence of the original ELLA expressions represented by the deterministic machines. The logical expressions are further decomposable into simpler regularly structured VCs, which are easier to prove automatically by an independent theorem-proving tool. Additional logical information can be included at the theorem-proving stage, by defining axioms, therefore allowing the introduction of application specific designer knowledge into the verification process. The complexity of the verification task may be reduced in this way by abstracting functions and representing their pertinent behaviour axiomatically. The VCs contain fragments of the original designs, and therefore provide high-level feedback for debugging purposes, if the designs prove to be inequivalent.

The development of the state evolution method and $\mathrm{VC}$ generation is only possible due to the nature of the semantic definition for ELLA, and the resulting objects: we have defined the semantics of ELLA at a high level, using a process algebraic approach which retains much of the original design structure. It would not have been possible to develop the state evolution/VCG verification method if the ELLA semantics were still represented by the low-level IOAs from $\$ 2.1$.

A detailed account of the derivation of the state evolution rule appears in [7], together with a proof of its soundness, with respect to strong bisimulation equivalence.

\section{Examples and Tool Implementation}

(1) 110-Detector An implementation of a simple boolean pattern detection design, taken from [19], is displayed by the schematics editor in the $E V E$, shown in Fig.1. This recognises ' 110 ' patterns in the input stream. This schematic is to be compared with the following specification:

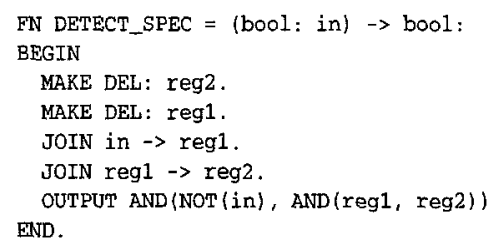

The schematic is translated into ELLA-text, the two designs are compiled into EPA deterministic machine forms, and two VCs are generated by the VCG; these can be proved automatically by PVS. We are able to abstract the logical functions and not expand their definitions in the VCG, since we can use directly the built-in boolean theory from PVS.

However, since the designs are small and only use boolean types, they can also be processed by our state-based compilation and verification tools. Even with the restricted 


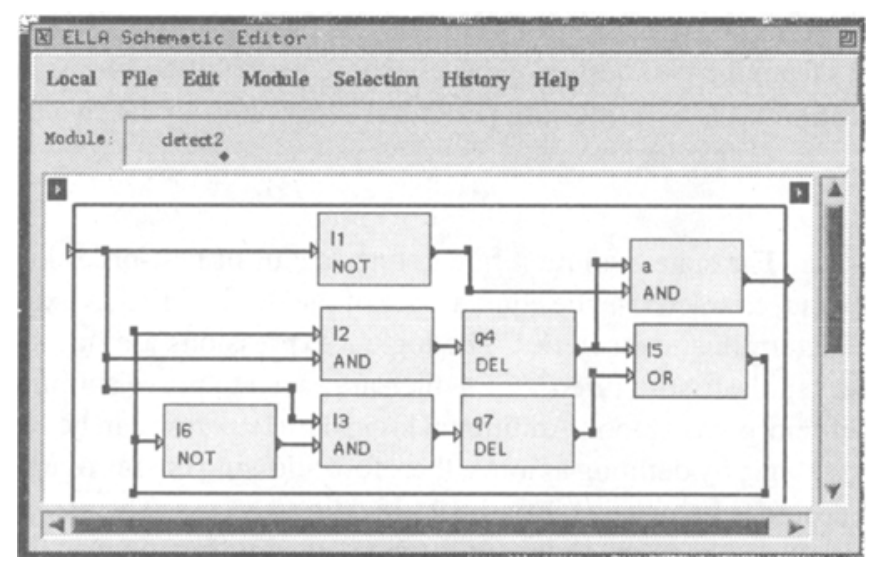

Fig. 1. 110-Detector Implementation

prototype implementations used, Table 2 below shows that the state-based approach is far quicker on this size of design. For small boolean designs of this nature, the overhead of using symbolic and theorem-proving methods for verification may therefore be too great, and standard state-based approaches may well be more efficient. Of course, any debugging feedback obtained from inequivalent designs using the state-based approach, might not be as useful as that provided by the high-level verification method.

(2) ALU Two implementations of a 16-bit ALU[18] are compared, one using a bit-slice structure, and the second using a behavioural structure. The ALU is controlled by a 5 bit word, and can perform addition, subtraction, shifting and logical operations on the input words. The designs are purely combinatorial and the BDD-based verification tool is ideally suited to analyse the designs. The internal variable ordering for the BDDs is of course crucial - the one used results in BDDs which are linear in the word size. Note that this design illustrates 'transition-explosion' for the state-based approach the single-state IOA would need $2^{38}$ transitions in order to represent each design.

(3) Integer Latch A latch design is now used to illustrate some of the benefits of using the state evolution method. The simple enabled integer latch design $P$, shown in Fig.2, also has a data-dependent control, where the latch is reset at the next time-step $t+1$ if it receives the data input value $i / 2000$ at time $t$. A second implementation $Q$ stores the integer in two halves, derived using mod and div operations. The VCG generates three VCs shown in Fig.3, resulting from the product of the two control structures. Note that the VCG has identified and discarded those VCs, generated for the merged machines, where the control branches have conflicting guard set expressions, using its set simplification rules. We need the following axiom to prove the second VC:

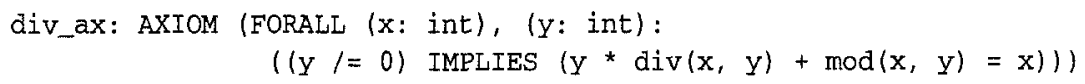




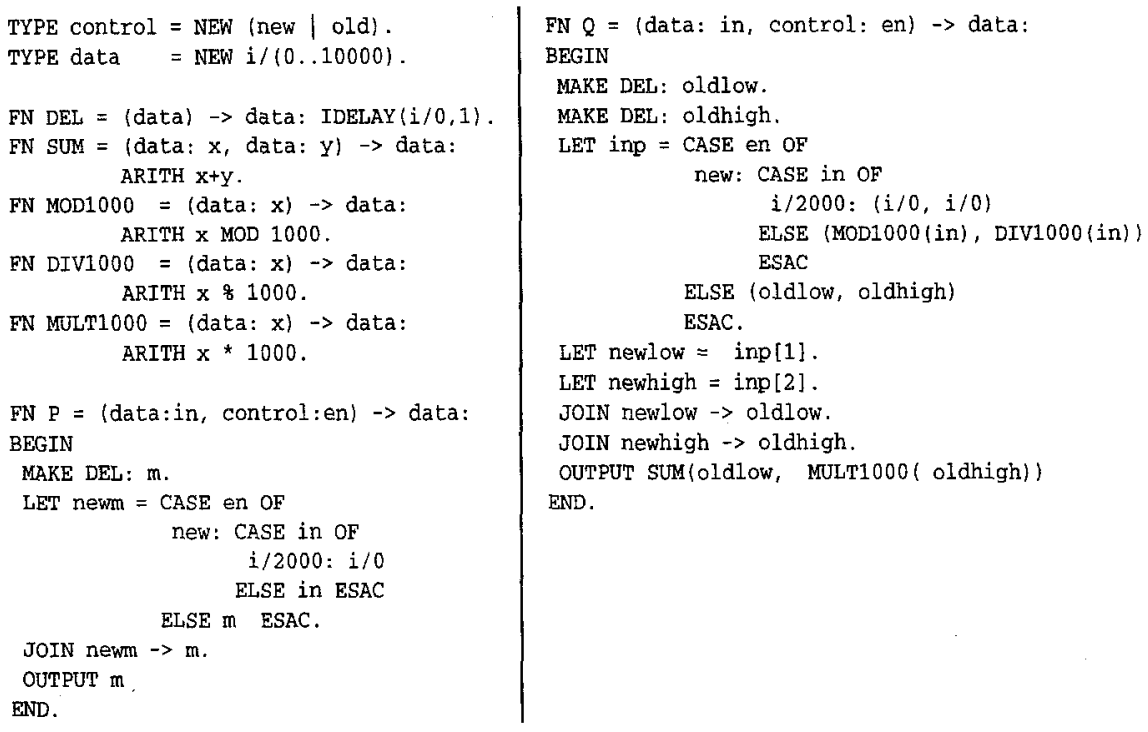

Fig. 2. Integer Latch Designs

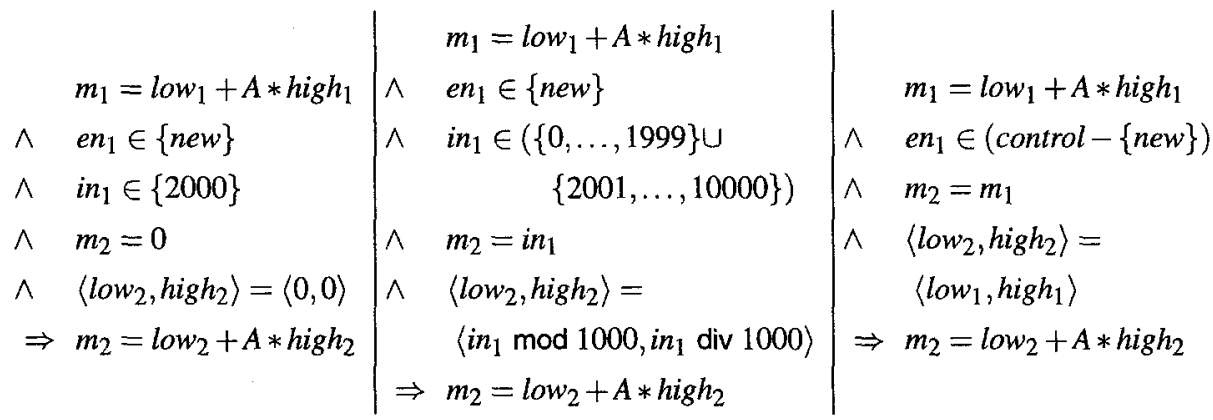

Fig. 3. VCs for Integer Latch

The axiom simply captures the designer's intuition when deriving the second implementation. With this axiom, all the VCs can be proved automatically in PVS. Note that, due to the integer arithmetic operations involved, even state-based verification methods using BDDs are likely to fail, since it will not be possible to find a BDD variable ordering leading to an efficient encoding of the problem.

This simple example highlights some of the advantages of using the state evolution approach:

- function abstraction is exploited during design verification, and designer information can be included at the theorem-proving stage via axioms, 
- VC simplification with non-trivial guard sets,

- dataset reduction and data/control separation - only expressions appearing as case selectors need to be analysed,

- designs can have different state-spaces; however the verification method can take advantage of similarities between the control structures of designs.

(4) FIR Filter Finally a slightly larger FIR filter design is given, similar to the TPCD'94 benchmark $1 S y s t{ }^{9}$, but defined at the integer level, as shown in Fig.4 and Fig.5. Design $Q$ is obtained from $P$ by performing a (manual) retiming, to increase overall circuit speed by reducing the clock cycle-time ${ }^{10}$. The two circuits each perform the following operation:

$$
y(t)=1 \cdot x(t-1)+2 \cdot x(t-2)+3 \cdot x(t-3)+4 \cdot x(t-4)+5 \cdot x(t-5)
$$

where $x(t)$ and $y(t)$ are the integer input and output values, respectively, at time $t$.

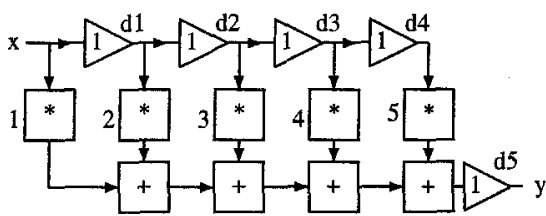

Fig. 4. FIR Filter Design $P$

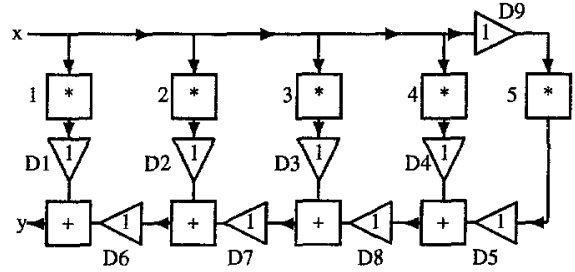

Fig. 5. FIR Filter Design $Q$

The VCG generates a single VC which simply contains a tautology as its conclusion, automatically provable using PVS - only the commutative/associative properties of integer multiplication and addition are required. Again, BDD-based methods are likely to fail here due to the integer arithmetic operations involved. Note that the complexity of the proof is not affected by the size of the input data or coefficients.

\subsection{Experimental Results}

Table 2 shows the times in seconds for compilation and verification of the example designs, with the $E V E$ running on a SparcStation-20, for the different verification tools included. An entry '-' in a column means that the particular tool was not applied to the design. Column IOA shows the total time for compilation of the two ELLA designs and

\footnotetext{
${ }^{9}$ The TPCD'94 description is obtained via ftp from goethe.ira.uka.de:/pub/tpcd94/benchmarks/1Sys to

${ }^{10}$ We thank Nick Ray (GEC Plessey Semiconductors, Swindon, UK) for suggesting this example.
} 


\begin{tabular}{|c||c||c|c|c|c|c|}
\hline \multicolumn{1}{|c||}{ Design } & \multicolumn{1}{|c||}{ IOA } & \multicolumn{5}{c|}{ Symbolic Verification } \\
\cline { 3 - 7 } & & Compile & VCG & Simplify & Num & PVS \\
\hline Detect & 0.87 & 6.1 & 3.9 & 2.6 & 2 & 44.0 \\
ALU & 13.1 & - & - & - & - & - \\
Latch & - & 4.7 & 12.9 & 13.1 & 4 & 11.1 \\
FIR & - & 17.5 & 336.2 & 443.4 & 2 & 228.9 \\
\hline
\end{tabular}

Table 2. Experimental Results

verification using trace-equivalence for Detect, or via BDDs for $A L U$. The state-based tools are only applied to relatively small boolean designs. The rest of the table contains details of the symbolic verification approach; Compile is the compilation time of the ELLA descriptions of the two designs into EPA and then into deterministic machines. $V C G$ is the VC generation time; Simplify is the time for further simplification of the resulting VCs (e.g. removing variable bindings), and converting into the PVS format. Num gives the total number of VCs output. Finally, PVS gives the total time spent verifying the VCs generated using PVS, running independently on a SparcStation-10.

\subsection{Tool Implementation}

The EVE contains conventional design tools such as design management, compiler, simulator and editors, as well as the novel formal analysis tools. It also includes an ELLAlevel transformation tool which can apply behaviour-preserving rules to an ELLA-text. The implementation of all tools is based on the formal semantics for ELLA. The environment has been implemented in Common Lisp by the ELLA Project partners, using LispWorks[12], which includes a GUI builder facilitating the development of the EVE user interface. The ELLA-to-EPA compiler together with the VCG automatically generate the set of VCs required for establishing the equivalence of two designs described in ELLA - the user only has to supply the number of steps $N$ for the state evolution rule $^{11}$. Of course, the designer must interact with the theorem-proving tool in order to prove the VCs. However, the VCG will have already performed extensive simplification of the VCs, and removed those that it finds to be valid. The regular first-order structure of the VCs facilitates the proof of those remaining, which can be largely automated with powerful theorem-proving tools such as PVS.

The 'layered' approach to defining the formal semantics is reflected in the structure of the Lisp code for the compiler, and has greatly assisted in its development. A term manipulation package called GT has been developed for efficient implementation of the

11 Note that the VCG could automatically generate a suitable value for $N$ by analysing the latency of the designs. If $N$ is set to a value too small for the particular designs, the outcome will simply be that the VCs are satisfiable but not (dis)provable - if this is the case, then the exercise can be repeated using a larger value of $N$. 
underlying data structures involved, supporting term-sharing, rewriting, and lazy substitution.

\section{Summary and Related Work}

We have described the formal semantic foundations required to support the development of a fully integrated hardware design environment for ELLA, containing a collection of formal verification support tools. The environment is designed to be easy to use; the tool set is fully integrated, at the level of its internal data-structures, facilitating development of a common look and feel for each tool interface. The tools are based on formal semantics, helping to ensure consistency of results, and significantly easing tool implementation. The user can interface with the environment via the ELLA HDL.

The formal semantics for ELLA was developed incrementally, and its layered style allows different back-end semantic representations to be generated, including IOAs and $E P A$ terms. Each representation supports a method for verification, which is applicable to different design types. In particular the EPA semantics supports truly symbolic verification techniques, based on deterministic machines. This provides efficient semiautomatic verification and high-level debugging feedback. We have obtained encouraging results from the initial prototype implementation of the environment, compilation and (symbolic) verification tools; the PVS system is currently used to validate the generated VCs.

Somewhat similar work, which aims to develop tractable formal verification methods, is reported in [19]. Here, logical expressions representing the complete verification problem are encoded in HOL[20]. The expression is then simplified sufficiently so that it can be automatically proven. This contrasts with our approach, of reformulating the verification problem so that a set of simpler (first-order) verification conditions characterising equivalence are generated from the outset, instead of having to simplify later at the theorem-proving stage. Also, in [21] the verification system is coupled with the CADENCE design system, although it is not clear that the logical formulae representing CADENCE designs are obtained via a formal semantics for the hardware description notations used in CADENCE.

A symbolic simulation technique for high-level processor verification is described in [22]. Here, a symbolic reachability graph is built for the product state machine of the two designs. However, this currently relies on syntactic identity in the functional equalities involved in order to establish design equivalence, which must be performed completely within the system, potentially leading to false negative results - the state evolution method described here does not have this restriction.

Our method bears some similarity to the approach used for establishing the correctness of the Viper microprocessor[23]. Here, a significant part of the work was involved in manually performing case analysis on the design representations, in order to extract the logical expressions required to show correctness. Only then could theorem-proving tools be used in the analysis.

Formal verification methods for hardware can be considered to use either state-based or 
theorem-proving techniques - our symbolic verification method has characteristics of both. State-based verification methods such as model-checking $[24,25]$, have traditionally been restricted by the problem of 'state-explosion'; however the capabilities of these techniques have been enhanced by the introduction of compact state-space encodings, namely $\mathrm{BDDs}[26,27,28]$. These have the advantage of offering an automatic verification approach, although the size of the BDDs depends critically on the ordering of the variables chosen, and the user may need to manually try out different orderings. Indeed for certain designs, the BDD size may explode exponentially whatever variable ordering is used. Moreover state-based methods are oblivious to data and control elements in a design, and are limited to finite-state systems, whereas our method essentially performs data abstraction automatically, leading to infinite state-space system verification. Of course, the number of VCs generated may be large, but this is due directly to the size of the control structures in the two designs.

In the second approach to verification, hardware systems are represented by collections of logical formulae and standard theorem-proving techniques are then applied in order to prove behavioural properties of the system. Our work shares many of the benefits of this approach, although it has additional advantages in terms of the potential ease of (automated) proof. The HOL system[20] introduced above is an extensive example of theorem-proving tools development, which can be applied to design verification. The PVS proof system utilised here, can be employed to model systems directly, and then to support the required proof task[29]. In general, these tools based on theorem-proving require extensive expertise in the use of the theorem-prover in order to operate them.

The underlying process algebraic approach of our method has its origins in the foundational works of CCS[4] and CSP[14]. There have recently emerged techniques for efficiently modelling systems; for example [30] describes a value-passing process algebra, where the structured actions consist of channels which can carry messages or data. Our work is also related to CIRCAL[15].

There have been other recent approaches to providing a formal semantics for ELLA. At Edinburgh, operational semantics are provided for simplified versions of ELLA in [31]. At Cambridge, a system has been developed to semantically embed ELLA into HOL [32], so that the HOL verification system and its associated tools can be employed.

In future work, the implementation of the tools will be refined, to take full advantage of term-sharing facilities provided by GT, and to enhance the VC simplification capabilities in the VCG. Interesting comparisons between different verification methods are now possible with the advantage of starting from the same ELLA source code - we can extend the suite of verification tools included, and attempt to interface with external tools as they are developed. We also aim to apply the technology to other HDLs, such as (synchronous) subsets of VHDL - the symbolic verification method can be applied to any designs whose behaviours can be represented as deterministic machines. The state evolution method will also be extended to the non-deterministic case. 


\section{References}

1. J. D. Morison and A. S. Clarke. ELLA2000: A Language for Electronic System Design. McGraw-Hill, 1993.

2. D. Schmidt. Denotational Semantics. Allyn and Bacon, 1986.

3. H. Barringer, G. Gough, T. Longshaw, B. Monahan, M. Peim, and A. Williams. Semantics and Verification for Boolean Kernel ELLA using IO Automata. In Advanced Research Workshop on Correct Hardware Design Methodologies (CHARME'91), Turin, Italy, May 1991.

4. R. Milner. Communication and Concurrency. Prentice Hall, 1989.

5. N. Shankar, S. Owre, and J. M. Rushby. The PVS Proof Checker: A Reference Manual (Beta Release). Technical report, Computer Science Laboratory, SRI International, March 1993.

6. H. Barringer, G. Gough, B. Monahan, and A. Williams. A Process Algebra Foundation for Reasoning about Core ELLA. Technical Report UMCS-94-12-1, University of Manchester, December 1994.

7. H. Barringer, G. Gough, B. Monahan, and A. Williams. A State Evolution Method for Verifying Hardware Systems. Technical Report UMCS-95-7-1, University of Manchester, July 1995. Also to be presented as a poster at CHARME'95, Frankfurt, Germany, October 1995.

8. Computer General Electronic Design, Chippenham, Wiltshire, United Kingdom. The ELLA Language Reference Manual, 5.1 edition, February 1991.

9. D. Coelho. The VHDL Handbook. Kluwer, 1989.

10. N.A. Lynch and M.R. Tuttle. An Introduction to Input/Output Automata. Technical Report MIT/LCS/TM-373, MIT, November 1988.

11. Formal Verification Support for ELLA. Technical report, DRA (Malvern), Harlequin Ltd. (Cambridge), and University of Manchester, Formal Verification Support for ELLA, IED project 4/1/1357, in JFIT Conference, December 1992.

12. Harlequin Ltd, Cambridge UK. Information about LispWorks is available on the World-Wide Web, URL: http: / /www . harlequin.co.uk/full/products/sp/lispworks . html.

13. H. Barringer, G. Gough, B. Monahan, and A. Williams. A Semantics for Core ELLA. Project Report D2.3b, Formal Verification Support for ELLA, IED Project 4/1/1357, University of Manchester, November 1992.

14. C.A.R. Hoare. Communicating Sequential Processes. Prentice-Hall, 1985.

15. G. A. McCaskill and G. Milne. Hardware Description and Verification Using the CircalSystem. Technical Report HDV-24-92, Department of Computer Science, University of Strathclyde, June 1992.

16. T. Bolognesi and E. Brinksma. Introduction to the ISO Specification Language LOTOS. Computer Networks and ISDN Systems, 14(1), 1987.

17. H. Barringer, G.D. Gough, B.Q. Monahan, and A. Williams. A Process Algebraic Semantics for Core ELLA. Technical Report UMCS-93-2-1, University of Manchester, November 1994.

18. H. Barringer, G. Gough, B. Monahan, and A. Williams. The ELLA Verification Environment: A Tutorial Introduction. Technical Report UMCS-94-12-2, University of Manchester, December 1994.

19. R. Kumar, K. Schneider, and T. Kropf. Structuring and Automating Hardware Proofs in a Higher-Order Theorem-Proving Environment. Formal Methods in System Design, 2, 1993.

20. M. Gordon and A. Pitts. The HOL logic and system (Chapter 3). In J. Bowen, editor, Towards Verified Systems, pages 49-70. Elsevier, October 1994.

21. T. Kropf, R. Kumar, and K. Schneider. Embedding Hardware Verification within a Commercial Design Framework. In G. Milne and L. Pierre, editors, Correct Hardware Design and Verification Methods (CHARME '93), LNCS, volume 683, pages 242-257, Arles, France, May 1993. Springer-Verlag. 
22. F. Corella. Automated High-Level Verification against Clocked Algorithmic Specifications. In D. Agnew, L. Claesen, and R. Camposano, editors, CHDL '93, Canada, 1993. NorthHolland.

23. A. Cohn. A Proof of Correctness of the Viper Microprocessor: The First Level. In G. Birtwistle and P. Subrahmanyam, editors, VLSI Specification, Verification and Synthesis, 1989.

24. E.M. Clarke, E.A. Emerson, and A.P. Sistla. Automatic Verification of finite state concurrent systems using Temporal Logic Specifications. ACM Transactions on Programming Languages and Systems, 8(2), 1986.

25. H. Barringer, M. Fisher, and G.D. Gough. Fair SMG and Linear Time Model Checking. In Sifakis [33].

26. R.E. Bryant. Graph-Based Algorithms for Boolean Function Manipulation. IEEE Transactions on Computers, C-35(8):677-691, 1986.

27. J.R. Burch, E.M. Clarke, and D.E. Long. Representing Circuits More Efficiently in Symbolic Model Checking. In DAC91, 1991.

28. O. Coudert, C. Berthet, and J-C. Madre. Verification of Synchronous Sequential Machines based on Symbolic Execution. In Sifakis [33].

29. N. Shankar. Verification of Real-Time Systems Using PVS. In C. Courcoubetis, editor, Computer Aided Verification, (CAV '93), LNCS, volume 697, Elounda, Greece, June 1993. Springer-Verlag.

30. M. Hennessy and H. Lin. Proof Systems for Message-Passing Process Algebras. Technical Report 5/93, University of Sussex, 1993.

31. K.G.W. Goossens. Embedding Hardware Description Languages in Proof Systems. PhD thesis, University of Edinburgh, 1992.

32. R. Boulton, M. Gordon, J. Herbert, and J. Van Tassel. The HOL Verification of ELLA Designs. Technical report, University of Cambridge Computer Laboratory, 1990.

33. J. Sifakis, editor. Automatic Verification Methods for Finite State Systems, LNCS, volume 407, Grenoble, France, 1989. Springer-Verlag. 